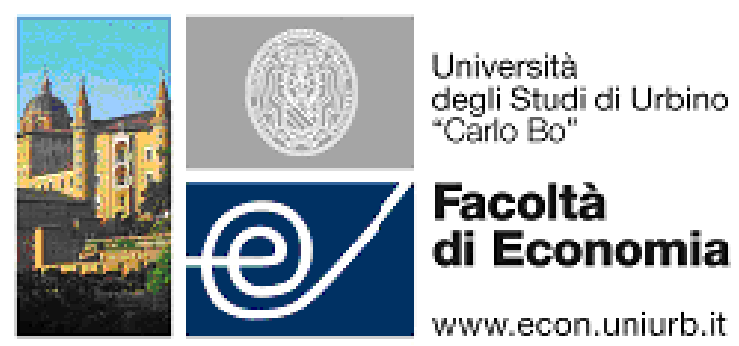

WP-EMS

Working Papers Series in Economics, Mathematics and Statistics

"Lead, Follow or Cooperate? Endogenous Timing \& Cooperation in Symmetric Duopoly Games"

- Marco Marini, (U. Urbino \& CREI, U. Roma III)

- Giorgio Rodano, (U. di Roma La Sapienza) 


\title{
LEAD, FOLLOW OR COOPERATE? ENDOGENOUS TIMING \& COOPERATION IN SYMMETRIC DUOPOLY GAMES
}

\author{
MARCO A. MARINI AND GIORGIO RODANO
}

\begin{abstract}
The aim of this paper is to extend Hamilton and Slutsky's (1990) endogenous timing game by including the possibility for players to cooperate. At an initial stage players are assumed to announce both their purpose to play early or late a given duopoly game as well as their intention to cooperate or not with their rival. The cooperation and timing formation rule is rather simple: when both players agree to cooperate and play with a given timing, they end up playing their actions coordinately and simultaneously. Otherwise, they play as singletons with the timing as prescribed by their own announcement. We check for the existence of a subgame perfect Nash equilibrium (in pure strategies) of such a cooperation-timing duopoly game. Two main results on the emergence of cooperation are provided. If players' actions in the symmetric duopoly game are strategic substitutes and there is no discount, cooperating early is a subgame perfect equilibrium of the extended timing-cooperation game. Conversely, cooperating late (at period two) represents an equilibrium when players' strategies are strategic complements. Other equilibria are also possible. Most importantly, our model shows that, in general, the success of cooperation is affected by the endogenous timing of the game. Moreover, the slope of players' best-replies appears crucial both for the success of cooperation as well as for the players' choice of sequencing their market actions.
\end{abstract}

Keywords: Endogenous Timing, Cooperation

JEL Classification Numbers: C70, C71, D23,D43.

Date: July 2011.

Corresponding author: Marco A. Marini, Department of Economics, Society \& Politics, Università di Urbino "Carlo Bo" and CREI, Università di Roma III. Address: via Saffi, 42, 60129, Urbino.(Italy). Tel. +39-0722-305557; Fax: +39-0722-305550. E-mail: marco.marini@uniurb.it.

Giorgio Rodano, Dipartimento di Informatica e Sistemistica "Antonio Ruberti", Università di Roma "La Sapienza". E-mail: giorgio.rodano@uniroma1.it.

We wish to thank Michael Kopel, Jacquelin Morgan, Maria Luisa Petit, Francesca Sanna Randaccio, George Zaccour and the participants at the MDEF Workshop in Urbino, SING7 in Paris, CREI Roma III, for their useful comments and discussions. 


\section{INTRODUCTION}

A wide number of papers have recently attempted to endogenize the timing of moves in a twoplayer duopoly model. In their seminal paper, Hamilton and Slutsky (1990) (HS, henceforth) consider an extensive form game (denoted extended game with observable delay) in which at a preplay stage two players (duopolists) decide independently whether to move early or late in the basic game (e.g., a duopoly quantity game). If both players announce the same timing (early, early) or (late, late), the basic game is played simultaneously. If the two players' time-announcements differ, the basic game is played sequentially, with the order of moves as announced by the players. HS's main results are that the two leader-follower configurations (with either order of play) constitute pure subgame perfect equilibria of the extended game only if at least one player's payoff as follower weakly dominates her corresponding payoff of the simultaneous game. In this case, in fact, neither the leader nor the follower have an incentive to deviate, inducing a simultaneous play. When, conversely, the payoff of a follower is lower than in the simultaneous case, the only pure strategy subgame Nash equilibrium prescribes that both players play simultaneously the basic game. As firstly shown by Gal-or (1985), Amir et al. (1999) and, most recently, by von Stengel (2010), in a symmetric duopoly in which some regularity conditions hold (mainly single-valued best-replies and payoffs monotone on rivals' actions) if players actions are strategic complements (and best-replies non decreasing) the follower's payoff dominates which of the leader (and therefore which of the simultaneous case). When instead actions are strategic substitutes (and best-replies non increasing) the opposite holds and a first-mover advantage exists. ${ }^{1}$

The aim of this paper is to extend Hamilton and Slutsky's (1990) endogenous timing game by including the possibility for players to cooperate. At a pre-play stage players are assumed to announce both their purpose to play early or late a given duopoly game as well as their intention to cooperate or not with the rival. The cooperation and timing formation rule is rather simple: when both players agree to cooperate and play with a given timing, they end up playing their actions coordinately and simultaneously. Otherwise, they play as singletons with the timing as prescribed by their own announcement. We check for the existence of a subgame perfect Nash equilibrium (in pure strategies) (SNE) of such a cooperation-timing duopoly game. Two main results on the emergence of cooperation are provided. If players' actions in the symmetric duopoly game are strategic substitutes and there is no discount, cooperating early is a SNE of the extended timing-cooperation game. Moreover, cooperating late is an equilibrium as long as there is not a too strong first-mover advantage which, in turn, depends on the slope of best-replies. In addition, as in HS (1990), the noncooperative simultaneous play is an equilibrium, although this outcome is Pareto-dominated for both players by the cooperative agreement. On the other hand, when players' actions are strategic complements, we show that cooperating late (at period two) always represents an equilibrium while, cooperating early (at period one) can, in some cases, be dominated by playing as follower the sequential game. As in HS (1990), sequential noncooperative configurations remain SNE and are also shown to be robust against joint deviations of the players. Most importantly, our model shows that, in general, the success of cooperation is affected by the endogenous timing of the game. Moreover, the slope of players' best-replies appears crucial both for the success of cooperation as well as for the players' choice of sequencing their market actions.

The paper is organized as follows. The next section introduces the game, and highlights how our model departs from HS's framework. Section 3 presents a few results on both the players' equilibrium strategies and the payoffs obtained in the duopoly game when actions are either strategic

\footnotetext{
${ }^{1}$ Without assuming players' actions as complements or substitutes, von Stengel (2010) proves that either the leader's payoff dominates that of the follower (which is also dominated by the simultaneous Nash) or, in turn, it is dominated by the follower's payoff. See also Dowrick (1985), Amir (1995), Amir and Grilo (1995), Amir, Grilo and Jin (1999) and Currarini and Marini (2003, 2004) for various leader-follower payoff comparisons among single or coalitions of players.
} 
substitutes or complements. To understand the forces at work, some examples of duopoly models under quantity and price competition are briefly presented. Section 4 concludes.

\section{The Model}

Let, at a pre-play stage denoted $t_{0}$, two players $i=1,2$ announce simultaneously both their intention to cooperate or not with the rival as well as the timing $\tau=\left(t_{1}, t_{2}\right)$ they intend to play the market game. Every player's announcement set $A_{i}$, for $i=1,2$ and $j \neq i$, can be defined as

$$
A_{i}=\left[\left(\{i, j\}, t_{1}\right),\left(\{i, j\}, t_{2}\right),\left(\{i\}, t_{1}\right),\left(\{i\}, t_{2}\right)\right],
$$

where the first two announcements possess a cooperative nature, while the last two correspond to the usual noncooperative timing choices (early, late) included in HS. As a result, players' announcement space $\left(A_{1} \times A_{2}\right)$ contains 16 different announcement profiles $a$ which, in turn, can induce the following set of timing-partitions $P(a)$ :

$$
\mathcal{P}=\left[\left(\{1,2\}^{t_{1}}\right),\left(\{1,2\}^{t_{2}}\right),\left(\{1\}^{t_{1}},\{2\}^{t_{1}}\right),\left(\{1\}^{t_{2}},\{2\}^{t_{2}}\right),\left(\{1\}^{t_{1}},\{2\}^{t_{2}}\right),\left(\{1\}^{t_{2}},\{2\}^{t_{1}}\right)\right] .
$$

Differently from HS (1990), here the two players are allowed to cooperate and form an alliance at period $t_{1}$ or $t_{2} .^{2}$

We assume that in order to form, an alliance endowed with a specific timing requires the unanimity of its members: when both players announce the intention to cooperate and to play an action at the same time, they will play cooperatively at the prescribed time; otherwise, they will behave as singletons with the timing as prescribed by their own announcement. Formally, for $i=1,2, j \neq i$ and $\tau=\left(t_{1}, t_{2}\right)$,

$$
\left\{\begin{array}{c}
P(a)=\{1,2\}^{\tau} \text { if } a_{i}=a_{j}=(\{i, j\}, \tau) \text { and } \\
P(a)=\left(\{i\}^{\tau_{i}},\{j\}^{\tau_{j}}\right) \text { if } a_{i} \neq a_{j}
\end{array}\right.
$$

The above rule prescribes that if players agree on both dimensions (timing and cooperation), they will end up playing cooperatively at a given time. Conversely, if just one player disagrees, either on cooperation or on timing, both players will play as singletons at their preferred time. ${ }^{3}$ Figure 1 represents the announcement game in strategic form. The payoff under cooperative, Stackelberg leader, Stackelberg follower and Nash simultaneous play are denoted, respectively, as $\pi^{c}, \pi^{\ell}, \pi^{f}$ and $\pi^{n}$.

\begin{tabular}{|c|c|c|c|c|}
\hline$a_{1} \backslash a_{2}$ & $\left(\{1,2\}, t_{1}\right)$ & $\left(\{1,2\}, t_{2}\right)$ & $\left(\{2\}, t_{1}\right)$ & $\left(\{2\}, t_{2}\right)$ \\
\hline$\left(\{1,2\}, t_{1}\right)$ & $\pi^{c}, \pi^{c}$ & $\pi^{\ell}, \pi^{f}$ & $\pi^{n}, \pi^{n}$ & $\pi^{\ell}, \pi^{f}$ \\
\hline$\left(\{1,2\}, t_{2}\right)$ & $\pi^{f}, \pi^{\ell}$ & $\pi^{c}, \pi^{c}$ & $\pi^{f}, \pi^{\ell}$ & $\pi^{n}, \pi^{n}$ \\
\hline$\left(\{1\}, t_{1}\right)$ & $\pi^{n}, \pi^{n}$ & $\pi^{\ell}, \pi^{f}$ & $\pi^{n}, \pi^{n}$ & $\pi^{\ell}, \pi^{f}$ \\
\hline$\left(\{1\}, t_{2}\right)$ & $\pi^{f}, \pi^{\ell}$ & $\pi^{n}, \pi^{n}$ & $\pi^{f}, \pi^{\ell}$ & $\pi^{n}, \pi^{n}$ \\
\hline
\end{tabular}

Figure 1 - The announcement game in strategic form.

\footnotetext{
${ }^{2}$ Later on we will show that, by letting players 'cooperating across time', i.e. sequencing their cooperative strategy with announcements such as $a=\left\{1^{t_{1}}, 2^{t_{2}}\right\}$ or $a=\left\{1^{t_{2}}, 2^{t_{1}}\right\}$, would not alter the basic results of the analysis.

${ }^{3}$ Note that when both players announce, say, $a_{i}=\left(\{i\}, t_{1}\right)$, thus, according to the unanimity rule, their announcement differ: player 1 has announced $a_{1}=\left(\{1\}, t_{1}\right)$ and player $\left.2 a_{2}=\left(\{2\}, t_{1}\right)\right)$ and, therefore, they end up playing noncooperatively as singletons within the partition $P(a)=\left(\{1\}^{\tau_{1}},\{2\}^{\tau_{1}}\right)$. The same occurs when their announcement is $a_{i}=\left(\{i\}, t_{2}\right)$. In a two player environment, the unanimity rule works as the pairwise stability concept (Jackson and Wolinsky, 1996), where a link forms if and only if there is the agreement of both players, while everyone can sever a link unilaterally.
} 
2.1. Cooperative vs. Noncooperative Duopoly Games. Once every player has made an announcement $a_{i} \in A_{i}$ and a timing-partition, denoted $P(a) \in \mathcal{P}$, has been induced on the set of players, they can decide their (cooperative or noncooperative) strategy with a timing prescribed by $P(a)$. Let the two players possess real-valued strategy sets $X_{i}=X \subset \mathbb{R}_{+}$. Let also their payoffs $\pi_{i}\left(x_{i}, x_{j}\right): X^{2} \rightarrow \mathbb{R}$ be symmetric, i.e. such that $\pi_{i}\left(x_{i}, x_{j}\right)=\pi_{j}\left(x_{j}, x_{i}\right)$ for every $\left(x_{i}, x_{j}\right) \in X^{2}$. We restrict players' payoffs to be positively or negatively monotone in the rival's strategy. We will talk, in turn, of positive (PE) or negative externalities (NE). Moreover, player $i$ 's strategies will be defined strategic complements (substitutes) if and only if the payoff $\pi_{i}\left(x_{i}, x_{j}\right)$ exhibits increasing (decreasing) differences in $\left(x_{i}, x_{j}\right) \in X^{2}$, i.e., if the sign of $\pi_{i}\left(x_{i}, x_{j}^{\prime}\right)-\pi_{i}\left(x_{i}, x_{j}^{\prime \prime}\right)$ is positive (negative) for every $x_{j}^{\prime \prime}>x_{j}^{\prime}$. When players' payoffs are smooth, increasing (decreasing) differences corresponds to $\frac{\partial^{2} \pi_{i}}{\partial x_{i} \partial x_{j}}>(<) 0$ (Topkis, 1998). ${ }^{4}$

We are now ready to define the behaviour of players in the different duopoly games as induced by the timing-partitions described in (2.2). When cooperation takes place and the grand coalition $P(a)=\{1,2\}^{\tau}$ forms, both players will be assumed to set cooperatively their strategies either at stage $\tau=t_{1}$ or $t_{2}$ as

$$
x\left(\{i, j\}^{\tau}\right)=x^{c^{\tau}}=\left(x_{i}^{c^{\tau}}, x_{j}^{c^{\tau}}\right)
$$

where, for every $i=1,2$ with $j \neq i$

$$
x_{i}^{c^{\tau}}=\arg \max _{x_{i}} \sum_{i=1,2} \pi_{i}\left(x_{i}, x_{j}\right) .
$$

The above formulation implicitly assumes that players possess transferable utilities. However, no side payments are allowed between players and, therefore, their allocation follows by the solution of the cooperative problem (2.4). In what follows, we will presume a symmetric cooperative solution, namely, $x_{i}^{c^{\tau}}=x_{j}^{c^{\tau}}$ and, as a result, $\pi_{i}\left(x_{i}^{c^{\tau}}, x_{j}^{c^{\tau}}\right)=\pi_{j}\left(x_{i}^{c^{\tau}}, x_{j}^{c^{\tau}}\right) .{ }^{5}$ We will show in Appendix that, for certain classes of games (such as games with players' strict quasiconcave payoff functions and convex strategy sets), the cooperative solution is necessarily symmetric. ${ }^{6}$ This solution can be interpreted, for instance, as the formation of a merger (or an alliance) making players' choices binding. Therefore, once an alliance has formed, both players are assumed to behave just as a single entity maximizing their joint payoff.

If instead players move simultaneously as singletons either at stage $\tau=t_{1}$ or $t_{2}$, they will be assumed to play à $l a$ Nash, and the equilibrium $x^{n^{\tau}}$ of the duopoly game (played simultaneously at stage $\tau$ ) can be defined as

$$
x\left(\{i\}^{\tau},\{j\}^{\tau}\right)=x^{n^{\tau}}=\left(x_{i}^{n^{\tau}}, x_{j}^{n^{\tau}}\right)
$$

where, for every $i, j=1,2$ and $j \neq i$

$$
x_{i}^{n^{\tau}}=\arg \max _{x_{i}} \pi_{i}\left(x_{i}, x_{j}\right) .
$$

By symmetry, if the simultaneous Nash equilibrium $x^{n^{\tau}}$ of the duopoly game is unique, it will be such that $x_{i}^{n^{\tau}}=x_{j}^{n^{\tau}}$.

Finally, if players decide to act sequentially, the relevant equilibrium profile will be a Stackelberg (subgame perfect Nash) equilibrium, i.e. the profile

$$
x\left(\{i\}^{t_{1}},\{j\}^{t_{2}}\right)=x^{s}=\left(x_{i}^{s}, r_{j}\left(x_{i}^{s}\right)\right)
$$

\footnotetext{
${ }^{4}$ We exclude, for simplicity, the case of weakly increasing (decreasing) differences, thus implying, that players' best-replies are either strictly increasing or strictly decreasing functions.

${ }^{5}$ Note that by allowing side-payments among players, the only strong Nash allocation would always be the cooperative one. The equal split allocation is not stricly needed to define a SNE of the game. In fact, even if players' shares of the joint payoff would differ, at least one of them could never get more than one-half of the joint payoff and then, the equal-split allocation would, again, constitute the relevant payoff to compare the equilibrium allocations.

${ }^{6}$ For alternative proofs of this fact, see also Currarini and Marini (2006)
} 
such that, for the leader (henceforth player $i$ )

$$
x_{i}^{s}=\arg \max _{x_{i}} \pi_{i}\left(x_{i}, r_{j}\left(x_{i}\right)\right)
$$

and, for the follower (henceforth player $j$ ), $r_{j}: X \rightarrow X$ such that

$$
r_{j}\left(x_{i}\right)=\arg \max _{x_{j}} \pi_{i}\left(x_{i}, x_{j}\right) .
$$

Note that for the whole game to be well defined, all equilibria (2.3), (2.5) and (2.6) have to exist and be unique. We will derive our main results under such presumption, so to keep the set of assumptions minimal. It can be observed that, if players' payoffs are assumed continuous and strictly quasiconcave, their best-replies are continuous and single-valued. If, in addition, players' strategy sets are compact and convex, a Nash simultaneous equilibrium $x^{n^{\tau}}$ exists by Brower's fixed-point theorem. The existence of a Stackelberg equilibrium $x^{s}$ requires both the continuity of leader's payoff over her action and a follower's continuous best-reply, thus implying that the leader faces a continuous maximization problem over a closed set. By the Weierstrass theorem, a maximizer for the leader exists. The existence of a cooperative equilibrium $x^{c^{\tau}}$ is ensured by a continuous joint payoff. The uniqueness of these three equlibria is, in general, a more demanding property that here, for the sake of simplicity, we just assume. ${ }^{7}$

To close the model formalities, we introduce two equilibrium concepts for the announcement game played at the pre-play stage $t_{0}$. The first is a simple Nash equilibrium concept.

Definition 1. (Nash equilibrium) A timing-partition $P(a) \in \mathcal{P}$ is a Nash equilibrium of the announcement game if and only if $P=P\left(a^{*}\right)$ for some $a^{*}$ with the following property:

$$
\pi_{i}\left(x\left(P\left(a^{*}\right)\right) \geq \pi_{i}\left(x\left(P\left(a_{i}^{\prime}, a_{j}^{*}\right)\right)\right.\right.
$$

for any $a_{i}^{\prime} \in A_{i}$ and $i=1,2$.

Formally, if a timing-partition $P=P\left(a^{*}\right)$ is a Nash equilibrium, the pair $\sigma^{*}=\left(a^{*}, x^{*}\right)$ will be a subgame perfect Nash equilibrium (SNE) of the whole game, with the only warning that, at the subgame in which players decide to cooperate, they behave as a single maximizing entity. ${ }^{8}$ In addition, we propose a second concept of solution, usually known as strong Nash equilibrium (Aumann, 1959).

Definition 2. (Strong Nash equilibrium) A timing-partition $P(a) \in \mathcal{P}$ is a strong Nash equilibrium of the announcement game if and only if $P=P(\bar{a})$ for some $\bar{a}$ with the following property:

there exists no alternative announcement $a^{\prime} \in\left(A_{1} \times A_{2}\right)$ such that

$$
\pi_{i}\left(x\left(P\left(a^{\prime}\right)\right) \geq \pi_{i}(x(P(\bar{a})),\right.
$$

for both player $i=1,2$ and

for at least one player.

$$
\pi_{i}\left(x \left(P\left(a^{\prime}\right)>\pi_{i}(x(P(\bar{a}))\right.\right.
$$

A strong Nash equilibrium $\bar{a}$ is thus both a Nash equilibrium and a Pareto-optimal strategy profile of the announcement game.

\footnotetext{
${ }^{7}$ The uniqueness of a symmetric cooperative solution can be proved using the strict quasiconcavity of players' payoffs See the Appendix.

${ }^{8}$ Formally, when the profile $a=\{1,2\}^{\tau}$ is selected in the announcement game, firms are assumed to merge and sign a binding agreement to play their cooperative action profile so that no defections are allowed. This is in the tradition of noncooperative coalition formation literature (see Ray and Vohra 1997, Bloch 2003, Yi 2003, Ray 2007, Marini 2008 for surveys).
} 


\section{Main Results}

3.1. Payoffs and Equilibrium Actions. It is well known that, in all symmetric duopoly games in which players possess payoff functions monotone in their rivals' actions and single-valued bestreplies, if their actions are strategic substitutes (and best-replies decreasing), their equilibrium payoff will respect the following inequality: ${ }^{9}$

$$
\pi^{\ell}>\pi^{n}>\pi^{f}
$$

where $\pi^{n}$ indicates each player's payoff at the simultaneous Nash equilibrium (2.5), while $\pi^{\ell}$ and $\pi^{f}$ denote the leader and follower's payoffs, respectively, at the subgame perfect Nash (Stackelberg) equilibrium of the sequential game (2.6). On the other hand, when players' actions are strategic complements (and best-replies increasing), it is obtained that ${ }^{10}$

$$
\pi^{f}>\pi^{\ell}>\pi^{n} .
$$

This means that, when (3.1) holds, since every player prefers to be leader and none wants to follow, in the endogenous timing game à la HS (1990) there exists a unique pure SNE in which players play simultaneously. If, conversely, (3.2) holds, both sequential (Stackelberg) equilibria are SPE, with either order of play among players.

Now, since we have expanded the pre-play players' strategy set as in (2.1), we need to check whether the cooperative payoff $\pi^{c}$ obtained by a player either at stage $t_{1}$ or $t_{2}$ under (2.3) is profitable when compared to noncooperative simultaneous or sequential payoffs. In what follows, we will present some general results that do not require in general the monotonicity of players' bestreplies (implied by the property of increasing or decreasing differences of their payoffs). However, for simplicity, we will characterize our main results for the two classes of duopoly games with actions that are, in turn, strategic complements or substitutes.

A first result will simply be based on (3.1)-(3.2) and on the Pareto-efficiency of the cooperative outcome.

Proposition 1. In all symmetric duopoly games in which players' strategies are strategic complements (substitutes) the payoff of a player under cooperation must be higher than the leader's (follower's) payoff in the sequential game, namely, $\pi^{c}>\pi^{\ell}\left(\pi^{c}>\pi^{f}\right)$.

Proof. Suppose by contradiction that, when the strategies of the duopoly game are strategic complements

$$
\pi^{c}<\pi^{\ell}
$$

and, therefore, using (3.1), that

$$
\pi^{c}<\pi^{\ell}<\pi^{f}
$$

By symmetry, it follows that

$$
2 \pi^{c}<\pi^{\ell}+\pi^{f}
$$

contradicting the efficiency of the cooperative profile $x^{c^{\tau}}$. Similarly, if the strategies are strategic complements and $\pi^{c}<\pi^{f}$, by $(3.2)$

$$
\pi^{c}<\pi^{f}<\pi^{\ell}
$$

and again

$$
2 \pi^{c}<\pi^{\ell}+\pi^{f}
$$

a contradiction.

\footnotetext{
${ }^{9}$ Here best-replies are strictly monotone and, therefore, Nash and Stackelberg equilibria differ, i.e, $x^{s} \neq x^{n}$. As a result, the cases such that $\pi^{\ell}=\pi^{n}$ and $\pi^{f}=\pi^{n}$ do not arise.

${ }^{10}$ In von Stengel (2010) the monotonicity of best-replies to prove these payoff rankings is dispensed for. Also our proofs do not require such feature of players' best-replies. However, for ease of exposition, we divide our analyis in duopoly games with actions that are, respectively, strategic complements and substitutes.
} 
Corollary 1. In all symmetric duopoly games in which players' actions are strategic complements (substitutes), the following ranking between different payoffs arises: $\pi^{c}>\pi^{\ell}>\pi^{n}\left(\pi^{c}>\pi^{n}>\pi^{f}\right)$.

Proof. This is trivially obtained combining symmetry, the results of Proposition 1 and the efficiency of the cooperative allocation.

However, to find out the SNE of our timing-cooperation game, a complete ranking of players' payoffs in all different duopoly (or monopoly) games is required. More specifically, when players' actions are strategic complements, we need to assess whether, in turn, $\pi^{c}>\pi^{f}$ or $\pi^{f}>\pi^{c}$ and, instead, when players' actions are strategic substitutes, whether $\pi^{c}>\pi^{\ell}$ or $\pi^{\ell}>\pi^{c}$. The next section considers first the class of duopoly games with actions that are strategic complements.

3.2. Actions Strategic Complements. This section introduces a number of results on the relationship between players' equilibrium actions and their payoffs in the different duopoly games.

Proposition 2. In all symmetric duopoly games in which players' actions are strategic complements and extermalities are negative (NE) (positive $(P E)$ ), if a leader at the Stackelberg equilibrium plays an action that is lower (higher) than at the cooperative equilibrium, namely, $x_{i}^{s}\left\langle x_{i}^{c^{\tau}}\left(x_{i}^{s}\right\rangle x_{i}^{c^{\tau}}\right)$, the following ranking between players' payoffs arises: $\pi^{f}>\pi^{c}>\pi^{\ell}>\pi^{n}$.

Proof. If, at the sequential equilibrium, player $i=1,2$ in the role of leader plays an action such that $x_{i}^{s}<x_{i}^{c^{\tau}}$ (under NE) and $x_{i}^{s}>x_{i}^{c^{\tau}}$ (under PE), thus for the follower

$$
\pi_{j}\left(x_{i}^{s}, r_{j}\left(x_{i}^{s}\right)\right) \geq \pi_{j}\left(x_{i}^{s}, x_{j}^{c^{\tau}}\right)>\pi_{j}\left(x_{i}^{c^{\tau}}, x_{j}^{c^{\tau}}\right),
$$

where the first inequality stems by the Nash property of best-reply $r_{j}($.$) and the second by the$ property of monotone externalities and the fact that $x_{i}^{c^{\tau}}>x_{i}^{s}$ under NE and $x_{i}^{c^{\tau}}<x_{i}^{s}$ under PE. Therefore, by symmetry, $\pi_{j}\left(x_{i}^{s}, r_{j}\left(x_{i}^{s}\right)\right)=\pi^{f}$ and $\pi_{j}\left(x_{i}^{c^{\tau}}, x_{j}^{c^{\tau}}\right)=\pi^{c}$ and integrating this result with those of Proposition 1, it follows that $\pi^{f}>\pi^{c}>\pi^{\ell}>\pi^{n}$. Example 1 below shows that this condition is only sufficient and, by no means, necessary.

The results of Proposition 2 are illustrated in figure 2 and 3.

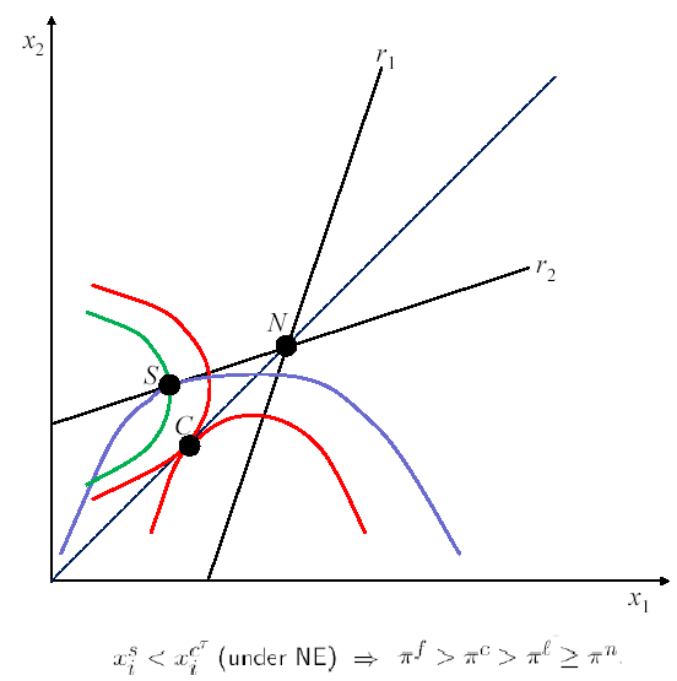

Figure 2 - Duopoly Game with strategic complements and negative externalities - Red = coop. isoprofits; Blu $=$ leader's isoprofit; Green $=$ follower's isoprofit. 


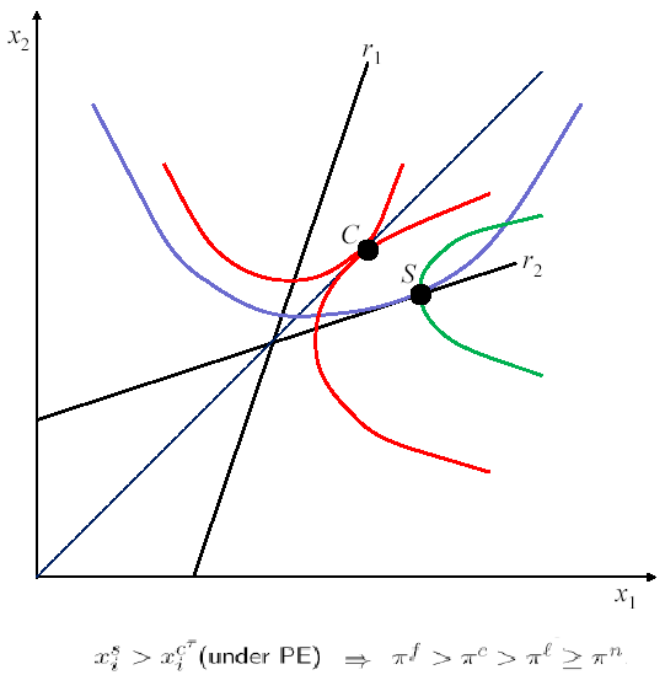

Figure 3 - Duopoly Game with strategic complements and positive externalities - Red = coop. isoprofits; $\mathrm{Blu}=$ leader's isoprofit; Green = follower's isoprofit.

The next proposition characterize the order of players' actions in the standard case in which a cooperative agreement gives a player a payoff higher than as a follower in the sequential game.

Proposition 3. In all symmetric duopoly games in which players' actions are strategic complements and players' payoffs at the cooperative equilibrium are higher than at a Stackelberg equilibrium, namely $\pi^{c}>\pi^{f}$, the following ranking between player equilibrium actions arises: $x_{i}^{n^{\tau}}>x_{j}^{s}>x_{i}^{s}>$ $x_{i}^{c^{\tau}}$ under $N E$ and $x_{i}^{c^{\tau}}>x_{i}^{s}>x_{j}^{s}>x_{i}^{n^{\tau}}$ under PE, where $x_{j}^{s}=r_{j}\left(x_{i}^{s}\right)$, for $i, j=1,2, j \neq i$ and $\tau=\left(t_{1}, t_{2}\right)$.

Proof. The fact that $x_{i}^{s}>x_{i}^{c^{\tau}}$ under negative externalities (NE) and $x_{i}^{s}<x_{i}^{c^{\tau}}$ under positive externalities (PE) whenever $\pi^{c}>\pi^{f}$ is directly implied by Proposition 2. The remaining inequalities are standard (see for instance Amir et al., 2000). In fact, taking a player $i$ in the role of leader,

$$
\pi_{i}\left(x_{i}^{s}, r_{j}\left(x_{i}^{s}\right)\right)>\pi_{i}\left(x_{i}^{n^{\tau}}, x_{j}^{n^{\tau}}\right) \geq \pi_{i}\left(x_{i}^{s}, x_{j}^{\tau}\right),
$$

where the first inequality holds since $x^{s} \neq x^{n^{\tau}}$ and the second by the Nash property of $x^{n^{\tau}}$. Thus, (3.3) directly implies that $r_{j}\left(x_{i}^{s}\right)=x_{j}^{s}<x_{j}^{n^{\tau}}$ under NE and $r_{j}\left(x_{i}^{s}\right)=x_{j}^{s}>x_{j}^{n^{\tau}}$ under PE. Since players' actions are strategic complements (and best-replies increasing), it also follows that $x_{i}^{s}<x_{i}^{n^{\tau}}$ under NE and $x_{i}^{s}>x_{i}^{n^{\tau}}$ under PE, since both Stackelberg and simultaneous Nash profiles lie along the increasing follower's best-reply. Finally, the fact that the isoprofit curves are concave (convex) under NE (PE) w.r.t. origin of the $X^{2}$ plan, implies that, at the point of tangency, $x_{j}^{s}>x_{i}^{s}$ under $\mathrm{NE}$ and $x_{j}^{s}<x_{i}^{s}$ under PE, and this concludes the proof.

Example 1. (Cournot with strategic complements) Let two firms face an inverse market demand function $P(Q)=(1+Q)^{-b}$, where $Q=\left(q_{1}+q_{2}\right)$ and $b>1$, with, for simplicity, production costs equal to zero. Every firm payoff is $\pi_{i}\left(q_{i}, Q\right)=(1+Q)^{-b} q_{i}, i=1,2$ and, thus, externalities are negative and quantities act as strategic complements, yielding increasing best-replies. Simple computations show that the equilibrium quantities are, respectively, $q_{i}^{n^{\tau}}=\frac{1}{b-2}, q_{i}^{s}=\frac{1}{b-1}, q_{j}^{s}=\frac{b}{(b-1)^{2}}$ and $q_{i}^{c^{\tau}}=\frac{1}{2(b-1)}$. Equilibrium payoffs are: $\pi^{n}=(b-2)^{-1}\left(\frac{b}{b-2}\right)^{-b}, \pi^{\ell}=(b-1)^{-1}\left(\frac{b^{2}}{(b-1)^{2}}\right)^{-b}, \pi^{f}$ $=(b-1)^{-2} b\left(\frac{b^{2}}{(b-1)^{2}}\right)^{-b}$ and $\pi^{c}=(2 b-2)^{-1}\left(\frac{b}{(b-1)}\right)^{-b}$. It is straightforward to see that, for $b \geq 2$, $q^{n^{\tau}}>q_{j}^{s}>q_{i}^{s}>q_{i}^{c^{\tau}}$ and $\pi^{c} \geq \pi^{f}>\pi^{\ell}>\pi^{n}$. Figure 1 illustrates this case. For $1<b<2$, there is 
a switch in players' payoffs and $\pi^{f}>\pi^{c}>\pi^{\ell}>\pi^{n} .{ }^{11}$ However, since $q_{i}^{s}=\frac{1}{b-1}$ and $q_{i}^{c^{\tau}}=\frac{1}{2(b-1)}$, it is easy to see that $q_{i}^{c^{\tau}}<q_{i}^{s}$ holds for any level of b. This proves that Proposition 2 is only sufficient and not necessary for $\pi^{f}>\pi^{c}$. This point is also illustrated graphically in Figures 4 and $5 .^{12}$

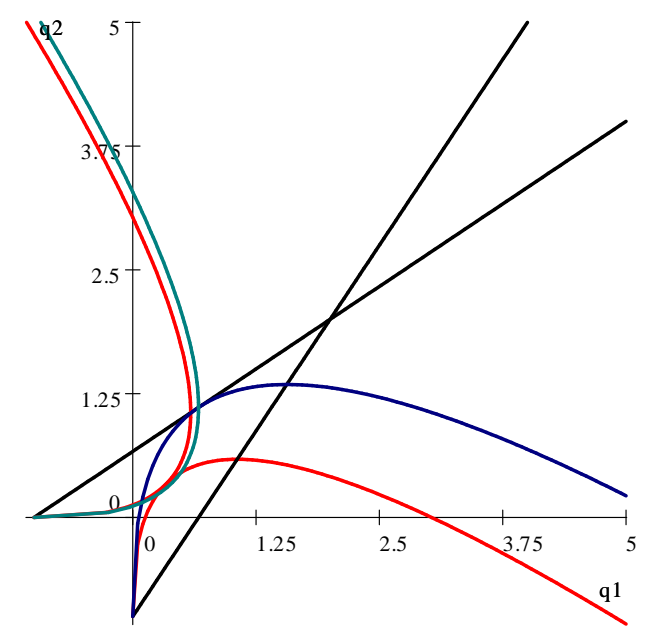

Figure 4 - Numerical example (Cournot with strategic complements) - Case $b=2.5$.Red $=$ coop. isoprofits; Blu = leader's isoprofit; Green = follower's isoprofit.

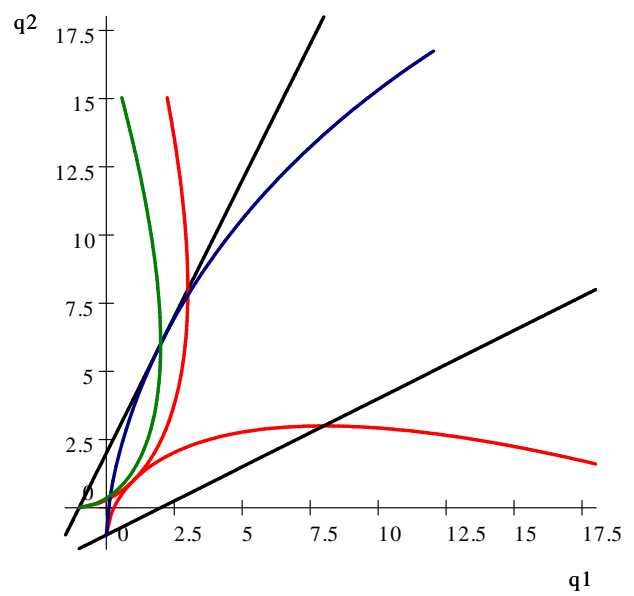

Figure 5 - Numerical example (Cournot with strategic complements) - Case $b=1.5$. Red $=$ coop. isoprofits; Blu = leader's isoprofit; Green = Ffollower's isoprofit.

The next proposition characterizes the relation between the actions of a leader and those of a follower at the Stackelberg equilibrium when the follower obtains a higher payoff than playing cooperatively. Together with Proposition 3, this result helps to see that, when actions are strategic complements (and best-replies increasing), the Stackelberg equilibrium will always lies below the 45 degree line when externalities are negative and above this line when externalities are positive (see, for instance, Figure 4 and 5 above).

\footnotetext{
${ }^{11}$ Note that, for $1<b<2$, firm best-replies are no contraction and for such range of parameters a simultaneous (Cournot) Nash equilibrium in pure strategies does not exist.

${ }^{12}$ As in the standard Cournot model, lower isoprofit curves correspond to higher profit quantity-combinations for firm 1, while the same holds for firm 2 isoprofit curves lying more on the left.
} 
Proposition 4. In all symmetric duopoly games in which players' actions are strategic complements and the follower's payoff at the Stackelberg equilibrium is higher than what obtained at a cooperative equilibrium, namely, $\pi^{f}>\pi^{c}$, the action of a follower is higher (lower) than the action of a leader under negative (positive) externalities, namely, $x_{j}^{s}>x_{i}^{s}$ under $N E$ and $x_{j}^{s}<x_{i}^{s}$ under PE, where $x_{j}^{s}=r_{j}\left(x_{i}^{s}\right)$.

Proof. Suppose not and $x_{j}^{s}<x_{i}^{s}$ under NE and $x_{j}^{s}>x_{i}^{s}$ under PE. Thus

$$
\pi_{j}\left(r_{j}\left(x_{i}^{s}\right), r_{j}\left(x_{i}^{s}\right)\right)>\pi_{j}\left(x_{i}^{s}, r_{j}\left(x_{i}^{s}\right)\right)>\pi_{j}\left(x_{i}^{c^{\tau}}, x_{j}^{c^{\tau}}\right)
$$

where the first inequality holds by monotone externalities and the second by the fact that $\pi^{f}>\pi^{c}$. Thus, since by symmetry $\pi_{j}(x)=\pi_{i}(x)$ for any $x$ such that $x_{i}=x_{j}$,

$$
\pi_{i}\left(r_{j}\left(x_{i}^{s}\right), r_{j}\left(x_{i}^{s}\right)\right)+\pi_{j}\left(r_{j}\left(x_{i}^{s}\right), r_{j}\left(x_{i}^{s}\right)\right)>\pi_{i}\left(x_{i}^{c^{\tau}}, x_{j}^{c^{\tau}}\right)+\pi_{j}\left(x_{i}^{c^{\tau}}, x_{j}^{c^{\tau}}\right)
$$

which contradicts the efficiency of $x^{c^{\tau}}$.

So far, the characterization of the various equilibria has shown that when actions are strategic complements two main equilibrium action-payoff configurations are possible. By Corollary 1 and Proposition 1, 2 and 3, under negative externalities (NE), when

$$
x^{n^{\tau}}>x_{j}^{s}>x_{i}^{s}>x_{i}^{c^{\tau}}
$$

both rankings of payoffs, either

$$
\pi^{c}>\pi^{f}>\pi^{\ell}>\pi^{n} \quad \text { or } \quad \pi^{f}>\pi^{c}>\pi^{\ell}>\pi^{n},
$$

are possible, while, when

$$
x^{n^{\tau}}>x_{j}^{s}>x_{i}^{c^{\tau}}>x_{i}^{s}
$$

thus, certainly,

$$
\pi^{f}>\pi^{c}>\pi^{\ell}>\pi^{n} .
$$

Conversely, under negative externalities (PE), when

$$
x_{i}^{c^{\tau}}>x_{i}^{s}>x_{j}^{s}>x_{i}^{n^{\tau}}
$$

both payoff-rankings

$$
\pi^{c}>\pi^{f}>\pi^{\ell}>\pi^{n} \text { or } \quad \pi^{f}>\pi^{c}>\pi^{\ell}>\pi^{n}
$$

can arise, while, when

$$
x_{i}^{s}>x_{i}^{c^{\tau}}>x_{j}^{s}>x_{i}^{n^{\tau}},
$$

thus, certainly,

$$
\pi^{f}>\pi^{c}>\pi^{\ell}>\pi^{n} .
$$

However, as the next example will illustrate, in a duopoly game with actions that are strategic complements (as in a classical price duopoly game), the case in which the follower's payoff overcomes the cooperative payoff rarely happens. When the follower's best reply is a contraction, it is unlikely that the leader's action is lower (higher) than its own cooperative choice under NE (PE) or, that the leader's action is low (high) enough under NE (PE) to make the follower better off than under cooperation. This property would normally require, in fact, a slope of best-replies largely greater than one under NE and largely lower than one under PE. 
Example 2. (Bertrand with differentiated products and strategic complements) Let every firm market demand be $q_{i}\left(p_{i}, p_{j}\right)=\left(1-p_{i}+\beta p_{j}\right)^{b}$, where $p_{i}$ and $p_{j}$ are the prices charged by the two firms respectively. Let also $b>0,0<\beta<1$, and costs normalized to zero for both firms. The payoffs are, therefore, simply given by $\pi_{i}\left(p_{i}, p_{j}\right)=\left(1-p_{i}+\beta p_{j}\right)^{b} p_{i}$. Note that for $\beta>0$, every firm payoff exhibit increasing differences and best-replies are increasing (prices work as strategic complements). Externalities are positive. The equilibrium payoffs when the game is played simultaneously and noncooperatively (at stage $t_{1}$ or $t_{2}$ ) is $\pi^{n}=(b-\beta+1)^{-1-b}(\beta-1+b-\beta+1)^{b}$, while, if the game is played sequentially à la Stackelberg, the leader obtains

$$
\pi^{\ell}=\left(b-\beta^{2}+1\right)^{-1}(b+1)^{-1}(b+\beta+1) \Delta,
$$

where

$$
\Delta=\left(\frac{\beta\left(b \beta^{2}-\beta-b \beta-b^{2}-2 b-1\right)}{(b+1)^{2}\left(\beta^{2}-b-1\right)}-\frac{b+\beta+1}{2 b+b^{2}-\beta^{2}-b \beta^{2}+1}+1\right)^{b},
$$

and the follower $\pi^{f}=(b+1)^{-2}\left(\beta^{2}-b-1\right)^{-1}\left(b \beta^{2}-\beta-b \beta-b^{2}-2 b-1\right) \Gamma$, where

$$
\Gamma=\left(\frac{\beta(b+\beta+1)}{2 b+b^{2}-\beta^{2}-b \beta^{2}+1}-\frac{\left(b \beta^{2}-\beta-b \beta-b^{2}-2 b-1\right)}{(b+1)^{2}\left(\beta^{2}-b-1\right)}+1\right)^{b} .
$$

Finally, when the two firms decide to merge, they jointly act as a single monopolist (playing either at stage $t_{1}$ or $t_{2}$ ) obtaining the equal-split payoff

$$
\pi^{c}=((\beta-1)+(b+1)(1-\beta))^{b}((b+1)(1-\beta))^{b-1} .
$$

Simple computations show that for all reasonable parameters values, $\pi^{c}>\pi^{f}>\pi^{\ell}>\pi^{n}$. Moreover, the following ranking for players' actions (prices) is obtained; $p_{i}^{c^{\tau}}>p_{i}^{s}>p_{j}^{s}>p_{i}^{n^{\tau}}$. Figure 6 depicts the case with $\beta=.5$ and $b=1$. No substantial changes in the ranking are obtained by manipulating the model parameters.

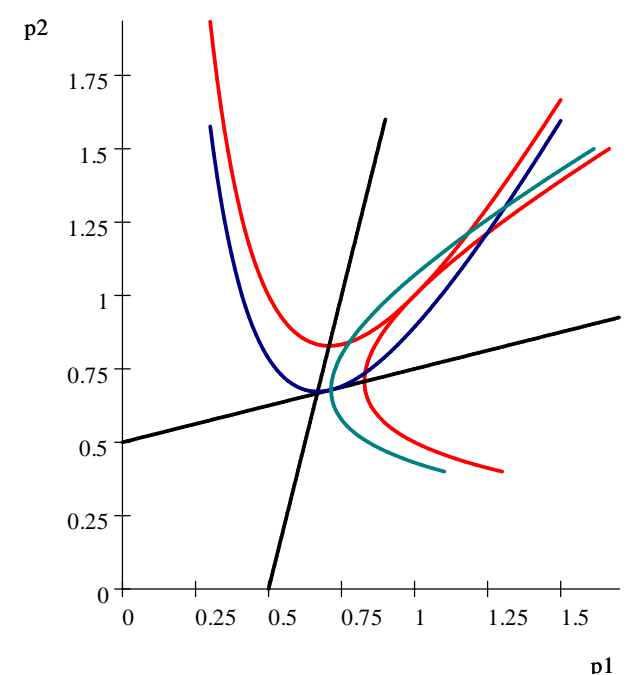

Figure 6 - Numerical example (Price competition with strategic complements) - Case $b=1$ and $\beta=0.5$. Red $=$ coop. isoprofits; Blu = leader's isoprofit; Green = follower's isoprofit.

We conclude the results of this section characterizing the Nash and strong Nash equilibria of the announcement game (and therefore SNE of the whole game) when the actions of the duopoly games are strategic complements. 
Proposition 5. In all symmetric duopoly games in which players' actions are strategic complements and the cooperative payoff is higher than the follower's payoff at the Stackelberg equilibrium, namely, $\pi^{c}>\pi^{f}$, (i) the set of Nash equilibrium timing-partitions of the announcement game is

$$
\mathcal{P}\left(a^{*}\right)=\left[\left(\{i, j\}^{t_{1}}\right),\left(\{i, j\}^{t_{2}}\right),\left(\{i\}^{t_{1}},\{j\}^{t_{2}}\right)\right],
$$

for $i, j=1,2$ and $j \neq i$, while the set of strong Nash timing-partitions is:

$$
\mathcal{P}(\bar{a})=\left[\left(\{i, j\}^{t_{1}}\right),\left(\{i, j\}^{t_{2}}\right)\right] .
$$

(ii) When, instead, $\pi^{f}>\pi^{c}$, the set of Nash and Strong Nash equilibrium timing-partitions is

$$
\mathcal{P}(\bar{a})=\mathcal{P}\left(a^{*}\right)=\left[\left(\{i, j\}^{t_{2}}\right),\left(\{i\}^{t_{1}},\{j\}^{t_{2}}\right)\right],
$$

Proof. (i) Under strategic complementarity and $\pi^{c}>\pi^{f}$, Proposition 1 has established the following ranking between players' payoffs:

$$
\pi^{c}>\pi^{f}>\pi^{\ell}>\pi^{n}
$$

Therefore, all cooperative announcements $a=(\{i, j\}, \tau)$ are Nash equilibria for any $\tau=\left(t_{1}, t_{2}\right)$, because no player can deviate profitably by announcing either $a_{i}^{\prime}=\left(\{i\}, t_{1}\right)$ or $a_{i}^{\prime \prime}=\left(\{i\}, t_{2}\right)$ inducing, in turn, the duopoly game played simultaneously or sequentially (see figure 1). As a result, any cooperative partition $P=\left(\{i, j\}^{\tau}\right)$ is part of a SNE of the whole game Note that in this case also "cooperating across time" (see footnote 1 ) would be a Nash equilibrium. Moreover, also the noncooperative sequential configuration $P=\left(\{i\}^{t_{1}},\{j\}^{t_{2}}\right)$ is a SNE since deviating unilaterally a player would end up playing simultaneously the duopoly game, obtaining, by (3.4), a lower payoff. The nonccoperative simultaneous play is not Pareto efficient and, therefore, is not a strong Nash. (ii) When $\pi^{f}>\pi^{c}$, the timing-partition $P(a)=\left(\{i, j\}^{t_{1}}\right)$ is no longer a Nash equilibrium since every player can deviate by announcing $a_{i}^{\prime}=\left(\{i\}, t_{2}\right)$ thus gaining a higher payoff as follower. The sequential timing-partition is both Nash and strong Nash stable, since by jointly announcing the grand coalition playing at time two would only improve the leader's and not the follower's payoff.

Proposition 4 helps to see that, when player actions are strategic complements, apart from the two sequential leader-follower configurations, also the two cooperative timing-partitions can be part of a SNE of the extended game. Moreover, cooperation at stage $t_{2}$ possesses robust stability properties, since is the only timing-partition to remain a strong Nash equilibrium in any circumstance.

3.3. Actions Strategic Substitutes. When player actions are strategic substitutes, there are only two possible rankings of players' equilibrium actions and payoffs under either negative or positive externalities. These will be characterized by the next proposition.

Proposition 6. (i) In all symmetric duopoly games in which players' actions are strategic substitutes the following rankings between equilibrium actions may arise: either $x_{i}^{s}>x_{i}^{n^{\tau}}>x_{j}^{s}>x_{i}^{c^{\tau}}$ or $x_{i}^{s}>x_{i}^{n^{\tau}}>x_{i}^{c^{\tau}}>x_{j}^{s}$ under $N E$ and $x_{j}^{s}>x_{i}^{c^{\tau}}>x_{i}^{n^{\tau}}>x_{i}^{s}$ or $x_{i}^{c^{\tau}}>x_{j}^{s}>x_{i}^{n^{\tau}}>x_{i}^{s}$ under PE, where $x_{j}^{s}=r_{j}\left(x_{i}^{s}\right), i, j=1,2$ and $\tau=\left(t_{1}, t_{2}\right)$. (ii) Moreover, only two alternative rankings among players' payoffs may arise: either $\pi^{c}>\pi^{\ell}>\pi^{n}>\pi^{f}$ or $\pi^{\ell}>\pi^{c}>\pi^{n}>\pi^{f}$.

Proof. (i) Similarly to Proposition 3 we can write, for the $i$-th player acting as leader,

$$
\pi_{i}\left(x_{i}^{s}, r_{j}\left(x_{i}^{s}\right)\right)>\pi_{i}\left(x_{i}^{n^{\tau}}, x_{j}^{n^{\tau}}\right) \geq \pi_{i}\left(x_{i}^{s}, x_{j}^{n^{\tau}}\right),
$$

where the first inequality stems from the fact that playing as leader strictly dominates the simultaneous Nash for every player and the second by the property of a Nash equilibrium. Therefore, it follows that $r_{j}\left(x_{i}^{s}\right)=x_{j}^{s}<x_{j}^{n^{\tau}}$ under NE and $r_{j}\left(x_{i}^{s}\right)=x_{j}^{s}>x_{j}^{n^{\tau}}$ under PE. Since actions are 
strategic substitutes, it follows that $x_{i}^{s}>x_{i}^{n^{\tau}}$ under NE and $x_{i}^{s}<x_{i}^{n^{\tau}}$ under PE, since both profiles $x^{s}$ and $x^{n^{\tau}}$ lie along the follower's decreasing best-reply. Thus

$$
x_{i}^{s}>x_{i}^{n^{\tau}}>x_{j}^{s}
$$

under NE and

$$
x_{j}^{s}>x_{i}^{n^{\tau}}>x_{i}^{s}
$$

under PE. By symmetry and payoff monotonicity coupled with the intrinsic efficiency of the cooperative strategy profile, we must also have that $x_{i}^{c^{\tau}}<x_{i}^{n^{\tau}}$ under NE and $x_{i}^{c^{\tau}}>x_{i}^{n^{\tau}}$ under PE. We remain, therefore, with the following two payoff inequalities:

either

$$
x_{i}^{s}>x_{i}^{n^{\tau}}>x_{j}^{s}>x_{i}^{c^{\tau}}
$$

or

$$
x_{i}^{s}>x_{i}^{n^{\tau}}>x_{i}^{c^{\tau}}>x_{j}^{s}
$$

under NE, and

$$
x_{j}^{s}>x_{i}^{c^{\tau}}>x_{i}^{n^{\tau}}>x_{i}^{s}
$$

or

$$
x_{i}^{c^{\tau}}>x_{j}^{s}>x_{i}^{n^{\tau}}>x_{i}^{s}
$$

under PE. Both cases may arise, as also the following example will illustrate.

(ii) From Proposition 1 we know that $\pi^{c}>\pi^{n}>\pi^{f}$ when actions are strategic substitutes. Given that $\pi^{\ell}>\pi^{n}$, it follows that either $\pi^{c}>\pi^{\ell}>\pi^{n}>\pi^{f}$ or $\pi^{\ell}>\pi^{c}>\pi^{n}>\pi^{f}$. The example below shows that both cases may arise. The superiority of leader's upon the cooperative payoff is more likely to occur when the follower's best-reply is very steep and, the ranking among players' actions are as in (3.5)-(3.7) above. However, such phenomenon may also arise when the other two rankings (3.6)-(3.8) are in place. The chance for the leader to expand (reduce) its action under $\mathrm{NE}(\mathrm{PE})$ and - along the follower's best-reply - to reduce (increase) her rival action, is the crucial factor for the leader to obtain a higher payoff than under a cooperative agreement. This happens when players' best-replies are (in absolute value) very steep.

Example 3. (Cournot Game with strategic substitutes) Let assume a market inverse demand given by $P(Q)=(1-Q)^{b}$, with $Q=\left(q_{1}+q_{2}\right)<1, b>0$ and no costs for both firms. The payoffs are therefore simply given by $\pi_{i}\left(q_{i}, Q\right)=(1-Q)^{b} q_{i}$, for $i=1,2$. Note that for $b>0$ payoffs exhibit decreasing differences and best-replies are decreasing (quantities work as strategic substitutes). Here externalities are negative. When the quantity game is played simultaneously and noncooperatively (at stage $t_{1}$ or $t_{2}$ ) a firm equilibrium payoff is $\pi^{n}=b^{b}(b+2)^{-(b+1)}$, while, if the game is played sequentially à la Stackelberg, the leader obtains $\pi^{\ell}=b^{2 b}(b+1)^{-(2 b+1)}$ and the follower $\pi^{f}=b^{(2 b+1)}(b+1)^{-(2 b+2)}$, respectively. Finally, when the two firms decide to merge, they jointly act as a single monopolist (playing either at stage one or two) thus obtaining the equal split payoff $\pi^{c}=b^{b} 2(b+1)^{-(b+1)}$. Simple computations show that for $b \geq 1$ (linear or convex demand) we obtain $\pi^{c} \geq \pi^{\ell}>\pi^{n}>\pi^{f}$ with the equal sign holding only for $b=1$. Figure 7 depicts the follower's best-reply and the two isoprofit curves, thus showing the described relation among players' payoffs. When, instead, $0<b<1$ (concave demand), $\pi^{\ell}>\pi^{c}>\pi^{n}>\pi^{f}$. Figure 8 illustrates this case. Figure 9 shows instead that $\pi^{\ell}>\pi^{c}$ arises when the inverse demand is highly concave (b very low). Moreover, the leader's payoff dominance upon the cooperative outcome may occur for a follower's quantity either lower or higher than the cooperative output. 


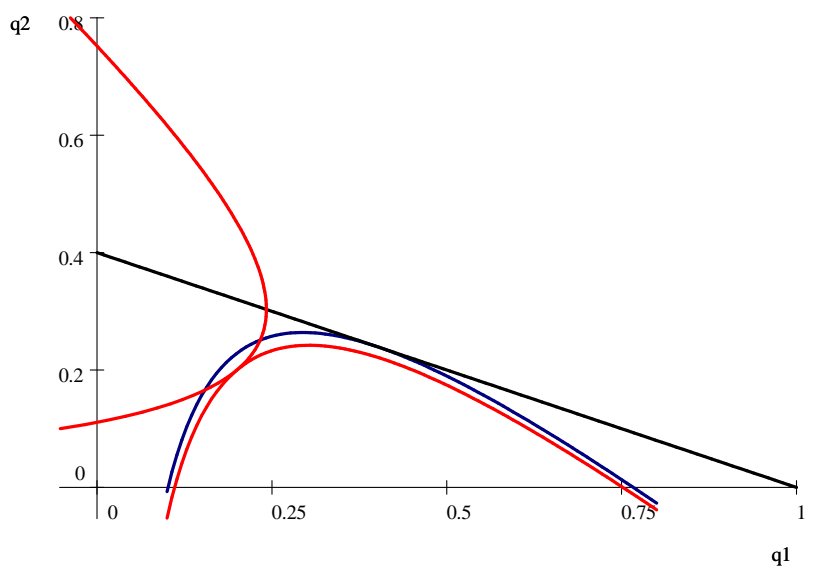

Figure 7 - Numerical example (Cournot with strategic substitutes) - Case $b \geq 1$. Red $=$ coop. isoprofits; $\mathrm{Blu}=$ leader's isoprofit.

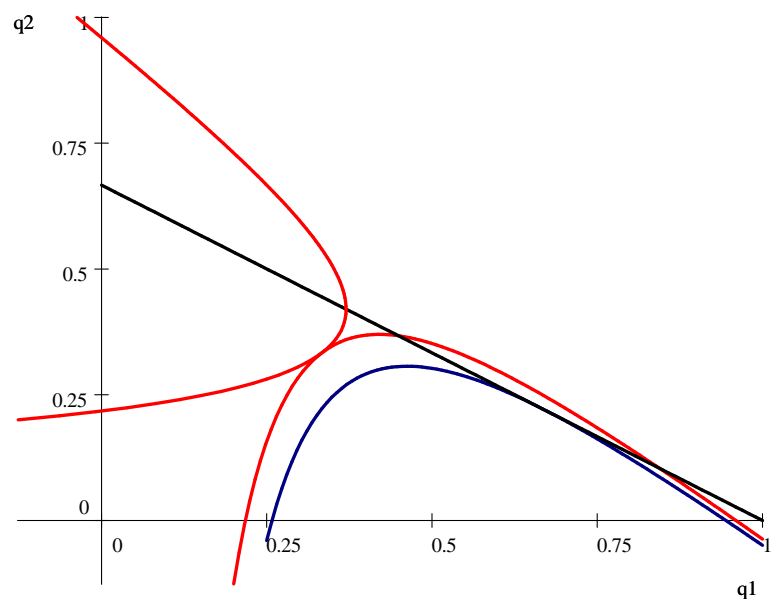

Figure 8 - Numerical example (Cournot with strategic substitutes) - Case $0<b<1$ Red $=$ coop. isoprofits; Blu = leader's isoprofit.

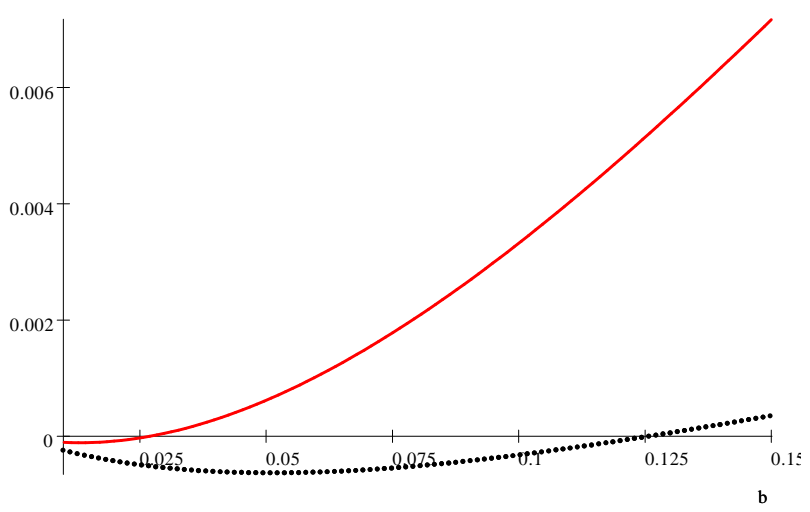

Figure 9 - Numerical example - Red line: $\left(x^{c^{t}}-x_{j}^{s}\right)=$ Coop. output - follower's output; Black dotted line: $\left(\pi^{c}-\pi^{\ell}\right)=$ Coop. Payoff - leader's payoff. $b=0.1, . ., 0.15$. 
We conclude this section with a full characterization of all timing-partitions which are in the set of Nash and strong Nash equilibria of the announcement game (and therefore are part of the SNE of the whole game).

Proposition 7. (ii) In all symmetric duopoly games in which players' actions are strategic substitutes and the cooperative payoff is higher than the leader's payoff at the Stackelberg equilibrium, namely, $\pi^{c}>\pi^{\ell}$, (i) the set of Nash equilibrium timing-partitions of the announcement game is

$$
\mathcal{P}\left(a^{*}\right)=\left[\left(\{i, j\}^{t_{1}}\right),\left(\{i, j\}^{t_{2}}\right),\left(\{i\}^{t_{1}},\{j\}^{t_{1}}\right)\right],
$$

for $i, j=1,2$ and $j \neq i$, while the set of strong Nash equilibrium timing-partitions is:

$$
\mathcal{P}(\bar{a})=\left[\left(\{i, j\}^{t_{1}}\right),\left(\{i, j\}^{t_{2}}\right)\right] .
$$

(ii) If, instead, $\pi^{\ell}>\pi^{c}$, the set of Nash equilibrium timing-partitions is:

$$
\mathcal{P}\left(a^{*}\right)=\left[\left(\{i, j\}^{t_{1}}\right),\left(\{i\}^{t_{1}},\{j\}^{t_{1}}\right)\right],
$$

while the set of strong Nash equilibrium timing-partitions is:

$$
\mathcal{P}(\bar{a})=\left[\left(\{i, j\}^{t_{1}}\right)\right] .
$$

Proof. (i) When actions are strategic substitutes and $\pi^{c}>\pi^{\ell}$, by Proposition 1 ,

$$
\pi^{c}>\pi^{\ell}>\pi^{n}>\pi^{f}
$$

Therefore, all cooperative announcements $a=(\{i, j\}, \tau)$ are Nash equilibria for any $\tau=\left(t_{1}, t_{2}\right)$, since no player $i=1,2$ can deviate profitably by announcing either $a_{i}^{\prime}=\left(\{i\}, t_{1}\right)$ or $a_{i}^{\prime \prime}=\left(\{i\}, t_{2}\right)$ inducing, respectively, the simultaneous or sequential duopoly game (see figure 1). Moreover, when

actions are strategic substitutes also the noncooperative simultaneous play $P=\left(\{i\}^{t_{1}},\{j\}^{t_{1}}\right)$ is a Nash equilibrium, just because, by deviating unilaterally with an alternative announcement $a_{i}^{\prime \prime}=\left(\{i\}, t_{2}\right)$, a player ends up playing sequentially as follower, thus obtaining a lower payoff. This simultaneous noncooperative equilibrium is not Pareto-efficient and therefore cannot be a strong Nash. (ii) When $\pi^{\ell}>\pi^{c}$, the cooperative timing-partition $P(a)=\left(\{i, j\}^{t_{2}}\right)$ is no longer a Nash equilibrium since every player can deviate by announcing $a_{i}^{\prime}=\left(\{i\}, t_{1}\right)$ and gaining a higher payoff as leader. Moreover, the simultaneous noncooperative partition cannot be improved upon by a player who deviates as follower. Finally, since cooperating at stage $t_{1}$ is both Nash stable and Pareto-efficient, this is the only strong Nash timing-partition of the announcement game.

\section{Concluding Remarks}

This paper constitutes a first attempt to connect two usually distinct issues concerning players' strategic interaction, one dealing with the endogenous timing of their actions, the other with their capacity to cooperate. The idea that players can coordinate their time of play when acting cooperatively, appears reasonable. Many meaningful social, economic and political examples witness the relevance of this issue. Our paper has introduced a new setup in which players can decide both the timing and the nature (cooperative or noncooperative) of their actions. We have shown that the type of the interaction among players in the strategic setting (duopoly game) plays an important role: when their actions are strategic complements (and best replies increasing), cooperation is more likely to be stable if deferred to the second stage. This is because no player can take advantage by deviating as leader (at stage one) expecting her rival reacting as follower. Similarly, no player can credibly object by inducing a noncooperative simultaneous play. Conversely, when the duopoly game is one of strategic substitutes (and best-replies decreasing), cooperation at stage one always emerges as a stable option. The reason is that no player can profitably deviate as follower expecting her rival to play as leader in a sequential game. Other equilibrium configurations may arise in the model, thus broadening the range of results which can be used to interpret strategically a number of economic and social situations. 


\section{Appendix}

Lemma 1. (Existence of a unique cooperative equilibrium) Let players' payoffs be continuous and stricly quasiconcave and their strategy set be compact and convex intervals $X_{i}=X \subset \mathbb{R}_{+}$. Then, there is a unique strategy profile $x^{c^{\tau}}=\arg \max _{x \in X^{2}} \sum_{i=1,2} \pi_{i}(x)$ and it is such that, $x_{1}^{c^{\tau}}=x_{2}^{c^{\tau}}$.

Proof. Compactness of each $X$ implies compactness of $X^{2}$. Continuity of each player's payoff $\pi_{i}(x)$ on $x$ implies the continuity of the social payoff function $\sum_{i=1,2} \pi_{i}(x)$. Existence of an efficient profile $x^{c^{\tau}} \in X^{2}$ directly follows from Weiestrass theorem. We prove that this strategy profile is symmetric.

Suppose $x_{i}^{c^{\tau}} \neq x_{j}^{c^{\tau}}$. By symmetry we can derive from $x^{c^{\tau}}$ a new vector $\widetilde{x}$ by permuting the strategies of players $i$ and $j$ such that

$$
\sum_{i=1,2} \pi_{i}(\widetilde{x})=\sum_{i=1,2} \pi_{i}\left(x^{c^{\tau}}\right)
$$

and hence, by the strict quasiconcavity of all $\pi_{i}(x)$, for all $\delta \in(0,1)$ we have that:

$$
\sum_{i=1,2} \pi_{i}\left(\delta \widetilde{x}+(1-\delta) x^{c^{\tau}}\right)>\sum_{i=1,2}\left(x^{c^{\tau}}\right)
$$

Since, by the convexity of $X$, the strategy vector $\left(\delta \widetilde{x}+(1-\delta) x^{c^{\tau}}\right) \in X^{2}$, we obtain a contradiction. Finally, by the strict quasiconcavity of both individual and social payoffs, the efficient profile $x^{c^{\tau}}$ can be easily proved to be unique.

\section{REFERENCES}

[1] Amir, R. (1995), Endogenous Timing in Two-player Games: A Counterexample. Games and Economic Behavior 9, 238-246.

[2] Amir, R., and I. Grilo (1999), Stackelberg versus Cournot equilibrium. Games and Economic Behavior 26, 1-21.

[3] Amir, R., I. Grilo, and J. Jin (1999), Demand-induced Endogenous Price Leadership. International Game Theory Review 1, 219-240.

[4] Amir, R. M. Amir and J. Jin (2000) Sequencing R\&D Decisions in a Two-period Duopoly with Spillovers, Economic Theory, 15(2), 297-317.

[5] Aumann, J.P (1959), Acceptable Points in General Cooperative n-person Games. In: Tucker, A.W., Luce, R.D. (eds.) Contributions to the Theory of Games IV. Princeton: Princeton University Press.

[6] Bloch, F. (2003) Coalition Formation in Games with Spillovers. in: Carraro C. (eds.) The Endogenous Formation of Economic Coalitions, Fondazione Eni Enrico Mattei Series on Economics and the Environment, Cheltenham, U.K. and Northampton, Mass.: Elgar.

[7] Bulow, J., J. Geneakoplos, and P. Klemperer (1985), Multimarket Oligopoly: Strategic Substitutes and Complements. Journal of Public Economics 93, 488-511.

[8] Currarini, S. and M., Marini, (2003), A Sequential Approach to the Characteristic Function and the Core in Games with Externalities, Sertel, M., Kara, A.(eds.), Advances in Economic Design. Springer Verlag, Berlin.

[9] Currarini, S. and M., Marini, (2004), A Conjectural Cooperative Equilibrium in Strategic Form Games, in Carraro C. , Fragnelli V. (eds.) Game Practise and the Environment. Kluwer Academic Press.

[10] Currarini, S. and M., Marini, (2006), Coalition Formation in Games without Synergies, International Game Theory Review, 8, 1, pp. 111-126.

[11] Dowrick, S. (1986), von Stackelberg and Cournot Duopoly: Choosing Roles. Rand Journal of Economics 17, 251-260.

[12] Gal-Or, E. (1985), First Mover and Second Mover Advantages. International Economic Review 26,649-653.

[13] Hamilton, J., and S. Slutsky (1990), Endogenous Timing in Duopoly Games: Stackelberg or Cournot Equilibria. Games and Economic Behavior 2, 29-46.

[14] Jackson, M.O., A. Wolinsky (1996) "A Strategic Model of Social and Economic Networks", Journal of Economic Theory, 71, 44-74.

[15] Marini, M. (2008), "Games of Coalition and Network Formation: a Survey", in Naizmada A. et al. (eds), Networks, Dynamics and Topology, London \& Berlin, Springer-Verlag.

[16] Ray, D. 2007. A Game-Theoretic Perspective on Coalition Formation. Oxford, U.K.: Oxford University Press. 
[17] Ray, D., and Vohra, R. 1997. Equilibrium Binding Agreements. Journal of Economic Theory 73: 30-78.

[18] Topkis, D.M. (1998), Supermodularity and Complementarity. Princeton: Princeton University Press.

[19] van Damme, E., and S. Hukens (1999), Endogenous Stackelberg Leadership, Games and Economic Behavior 28, $105-129$.

[20] van Damme, E., and S. Hurkens (2004), Endogenous Price Leadership, Games and Economic Behavior, 47, $404-420$.

[21] von Stengel, B. (2010), Follower Payoffs in Symmetric Duopoly Games, Games and Economic Behavior, 69, $512-516$.

[22] von Stengel, B., and S. Zamir (2004), Leadership with Commitment to Mixed Strategies.Research Report LSECDAM-2004-01, London School of Economics.

[23] Yi, S. S., (2003), The Endogenous Formation of Economic Coalitions: the Partition Function Approach, in C. Carraro (ed.). The endogenous formation of economic coalitions. Fondazione Eni Enrico Mattei Series on Economics and the Environment, Cheltenham, U.K. and Northampton, Mass.: Elgar. 